\title{
ESTIMACIÓN DE FACTORES DE EMISIÓN DE MATERIAL PARTICULADO RESUSPENDIDO ANTES, DURANTE Y DESPUÉS DE LA PAVIMENTACIÓN DE UNA VÍA EN BOGOTÁ
}

\section{ESTIMATION OF RESUSPENDED DUST EMISSION FACTORS BEFORE, DURING AND AFTER ROAD PAVING PROCESS IN BOGOTA}

\author{
Juan Felipe Méndez Espinosa',2, Laura Catalina Pinto Herrera², Boris René Galvis Remolina³, \\ Jorge E. Pachón ${ }^{4}$
}

Fecha de recepción: 29 de marzo de 2016

Fecha de revisión: 21 de junio de 2016

Fecha de aprobación: 26 de agosto de 2016

Referencia: J. F. Méndez Espinosa, L. C. Pinto Herrera, B. R. Galvis Remolina, J.E. Pachón (2017). Estimación de factores de emisión de material particulado resuspendido antes, durante y después de la pavimentación de una vía en Bogotá. Ciencia e Ingeniería Neogranadina, 27 (1), pp. 43-60, D0l: http://dx.doi.org/10.18359/rcin.1797

\section{RESUMEN}

En Bogotá, estudios previos han mostrado que las emisiones de material resuspendido constituyen una parte sustancial del inventario de emisiones e impactan de manera importante la calidad del aire de la ciudad. Esta investigación estimó los factores de emisión (FE) de material particulado resuspendido antes, durante y después de la pavimentación de la vía principal del barrio Caracolí, en la localidad de Ciudad Bolívar, por parte de la Unidad de Mantenimiento Vial del Distrito (UMV). Conjuntamente, se midió el impacto de la pavimentación en la calidad del aire.

1. Ingeniero Ambiental y Sanitario, Profesor Facultad de Ingeniería Ambiental, Universidad Santo Tomás, Villavicencio - Colombia, juanmendeze@ usantotomas.edu.co

2 Asistente de Investigación Centro Lasallista de Investigación y Modelación Ambiental CLIMA, Universidad de la Salle, Bogotá - Colombia, Ipinto1712@gmail.com

3 Ingeniero Químico. Magíster en Ingeniería Ambiental. Doctor en Ingeniería Ambiental, Profesor Asociado de la Facultad de Ingeniería de la Universidad de la Salle, Bogotá - Colombia, bgalvis@unisalle.edu.co

4 Autor de correspondencia. Director Centro Lasallista de Investigación y Modelación Ambiental CLIMA, Universidad de la Salle, Bogotá - Colombia, clima@lasalle.edu.co 
La valoración del impacto y estimación experimental de FE de polvo resuspendido se obtuvo a partir de mediciones y un análisis estadístico experimental entre concentraciones de $\mathrm{PM}_{10^{\prime}} \mathrm{PM}_{2.5^{\prime}}$ BC y variables meteorológicas al lado de la vía, junto con la aplicación de los modelos de dispersión SCREEN3 y AERMOD. Los factores de emisión estimados para vía no pavimentada fueron

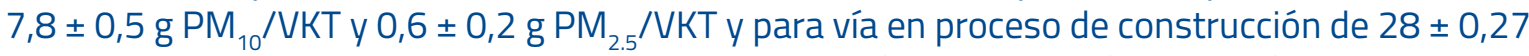
$\mu \mathrm{g} \mathrm{PM}_{10} / \mathrm{m}^{2 *}$ s y $11 \pm 0,13 \mu \mathrm{g} \mathrm{PM}_{2.5} / \mathrm{m}^{2 *} \mathrm{~s}$. La modelación de dispersión atmosférica de material particulado resuspendido mostró una reducción del área de impacto en aproximadamente $1 \mathrm{~km}$ y más de un $95 \%$ en concentración.

Palabras clave: modelación en calidad del aire, resuspensión de material particulado, pavimentación, factores de emisión.

\section{ABSTRACT}

Previous studies in Bogotá have shown that emissions of resuspended particulate matter constitute a significant part of the emissions inventory and have considerable impact on air quality. This research estimates resuspended dust emission factors before, during and after the pavement process of a secondary road in Bogota. The estimation of resuspended dust emission factors was obtained from measurements and data analysis of $\mathrm{PM}_{10^{\prime}} \mathrm{PM}_{2.5}$ and $\mathrm{BC}$ concentrations and meteorological variables recorded near the road. Later, SCREEN3 and AERMOD models were applied to estimate emission factors. The estimated emission factors for unpaved road were 7.8 $\pm 0.5 \mathrm{~g} \mathrm{PM}_{10} / \mathrm{VKT}$ and $0.6 \pm 0.2 \mathrm{gPM}_{2.5} /$ VKT respectively, and for road construction of $28 \pm 0,27$ $\mu \mathrm{g} \mathrm{PM}_{10} / \mathrm{m}^{2 *} \mathrm{~s}$ and $11 \pm 0,13 \mu \mathrm{g} \mathrm{PM}_{2.5} / \mathrm{m}^{2 *} \mathrm{~s}$. The atmospheric dispersion modeling of resuspended particulate matter showed a reduction of the impact area in roughly $1 \mathrm{~km}$ and more than $95 \%$ over concentration.

Keywords: air quality modeling, resuspension of particulate matter, paving, emission factors.

\section{INTRODUCCIÓN}

Una de cada ocho muertes en el mundo está asociada a la mala calidad del aire [1], sobre todo de grupos vulnerables como niños y ancianos, expuestos al material particulado (PM) [1-2]. En Colombia, el material particulado es uno de los contaminantes de mayor preocupación, dado el número de excedencias al año sobre la norma nacional [3] en ciudades como Bogotá, donde proviene principalmente de la resuspensión de material particulado en vías despavimentadas y en menor proporción pavimentadas [4]. Por su parte en Santiago de Chile el polvo resuspendido ha representado más de un 60\% del inventario de $\mathrm{PM}_{10}$ desde 1998 [5], actualmente si bien sigue siendo una fuente dominante de $\mathrm{PM}_{10}$, su cálculo actual presenta inconsistencias en la información [6] o posibles sobrestimaciones como en el caso de Bogotá [4]. En otras partes del mundo como Beijing se ha encontrado que la resuspensión es una de las fuentes que más aporta a la contaminación atmosférica, como también 
se presenta en España con un $60 \%$ de la carga de material particulado [4].

El PM resuspendido emitido en vías no pavimentadas es generado por procesos erosivos y/o meteorológicos o por el tránsito vehicular, el cual causa arrastre de polvo por vórtices verticales (resultado de la compresión y expansión de masas de aire), producción de material por desgaste de frenos y neumáticos (aportando a procesos abrasivos), y arrastre superficial con saltación geológica, siendo esta última, aplicable para partículas mayores a 10 micrómetros [7]. Investigaciones previas en Estados Unidos [7-10] han encontrado que las medidas de control más eficientes de remoción de material particulado susceptible de suspenderse son la pavimentación y la estabilización química, que permiten reducir sus emisiones aproximadamente hasta en un $90 \%$.

Conjuntamente se generaron factores de emisión de polvo resuspendido, a partir de mediciones y análisis estadístico-experimentales de concentraciones de material particulado y variables meteorológicas al lado de la vía, junto con la aplicación de modelos atmosféricos de dispersión (Técnica top-Down) acorde a lo establecido por Orozco et al. [11], facilitando a las autoridades gubernamentales e investigadores, realizar una mejor estimación de emisiones de polvo resuspendido en Bogotá y en zonas con condiciones geográficas y climatológicas similares, para posteriormente ser utilizadas en investigaciones aplicadas como la generación de inventarios de emisión.

En Bogotá, la Unidad de Planeación Zonal /smael Perdomo, en la que se encuentra el Barrio Caracolí en la localidad de Ciudad Bolívar, es catalogada según Decreto 623/2011, como área-fuente de contaminación Clase I por
$\mathrm{PM}_{10}$, por lo que se hace imperativo según la Resolución 601/2006, la ejecución de programas que contribuyan a la reducción y control del material particulado. Dentro del programa del Distrito Capital "Vías para superar la Segregación", la Unidad de Manteamiento Vial (UMV) estableció la intervención de una vía arterial del barrio Caracolí. Este programa ofreció la oportunidad de evaluar las concentraciones de $\mathrm{PM}_{10}$ y $\mathrm{PM}_{2.5}$ antes, durante y después del proceso de pavimentación, con el fin de medir el impacto en la calidad del aire que tiene la pavimentación de vías teniendo en cuenta la influencia de las condiciones meteorológicas sobre la resuspensión de PM; asimismo se estimaron experimentalmente factores de emisión de polvo resuspendido, a partir de mediciones en campo y modelos de dispersión (SCREEN3 y AERMOD) [12], aplicando el método desarrollado por Orozco et al. [11].

\section{MÉTODOS Y MATERIALES}

Se compararon los valores de tendencia central de material particulado por fuente (resuspensión, por combustión y no definida), obtenidos a partir de monitoreos automáticos de $\mathrm{PM}_{10}, \mathrm{PM}_{2.5}$ y $\mathrm{BC}$ a microescala antes, durante y después de la pavimentación. La determinación de la fuente de $\mathrm{PM}_{10}-\mathrm{PM}_{2.5}$ se obtuvo a partir del análisis estadístico-experimental de las concentraciones de contaminantes citados. Esto permitió valorar cuantitativamente el impacto de la pavimentación.

Se estimaron los FE a través del análisis estadístico-experimental, que incluyó la eliminación de las concentraciones de fondo mediante el análisis de vientos abajo y vientos arriba de la fuente, junto con la corrida inversa de AERMOD y SCREEN3. Las variables meteorológi- 
cas para la corrida de los modelos se obtuvieron a partir de mediciones en campo durante el monitoreo de contaminantes.

\subsection{Ejecución de campañas de monitoreo y área de estudio}

Se ejecutaron tres campañas de monitoreo de microescala a orillas de la vía (Kerbside) en la Calle 76 A sur, entre Carrera 73H y Carrera 73 HBIS del barrio Caracolí (Figura 1), antes, durante y después de su pavimentación. Se tuvieron en cuenta los criterios de localización (Figura 2) establecidos en el protocolo para el monitoreo y seguimiento de la calidad del aire de Bogotá [13] y el método EPA IO-2.1 [14], con el fin de obtener las concentraciones más representativas de la vía, asociadas a resuspensión.

Se usó el monitor DustTrak DRX 8533 (TSI, Shoreview MN) para la medición fotométrica en tiempo real de concentraciones de PM cada 10 segundos (flujo total de $3 \mathrm{~L} / \mathrm{min}$ con $\pm 5 \%$ de flujo de exactitud según norma ISO 12103-1), un aetalometro microAeth ${ }^{\circledR}$ integrado Modelo AE51 (Aethlabs, San Francisco CA) para la medición de concentraciones cada 10 segundos de carbono negro (BC) (flujo de 150 ml/min). Así mismo, se

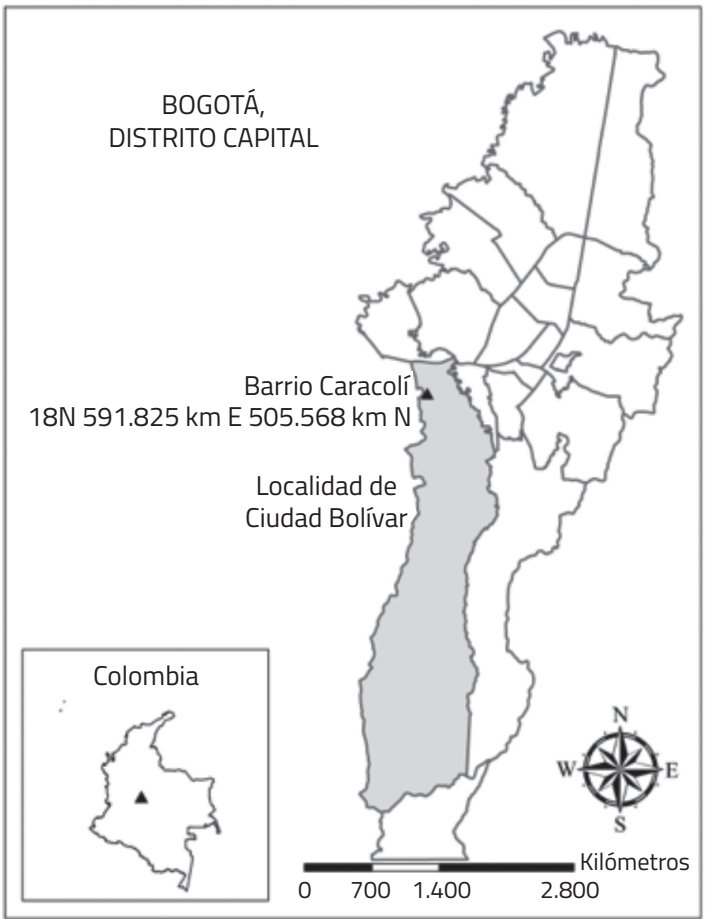

Figura 1. Ubicación geográfica de Caracolí - Ciudad Bolívar.

Fuente: Elaboración propia a partir de ArcGis. 


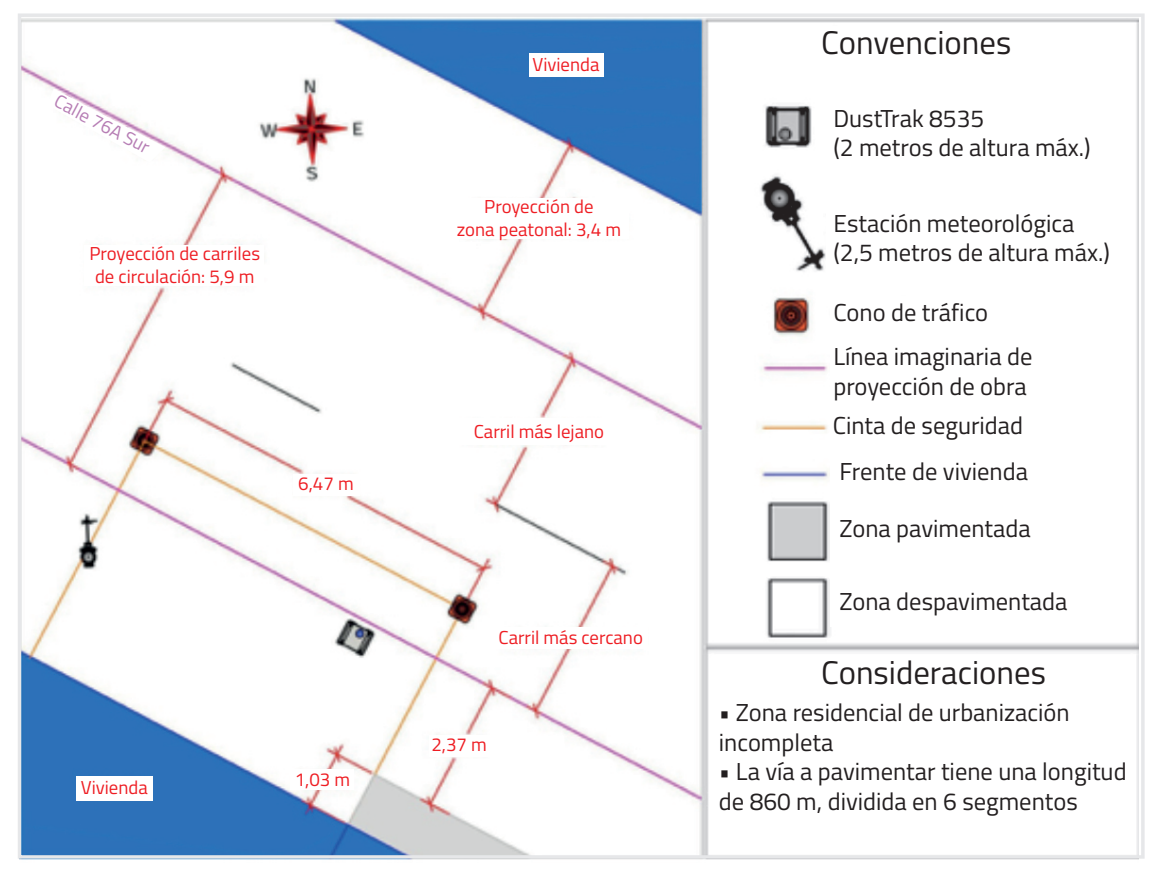

Figura 2. Establecimiento de punto de monitoreo y proyección de intervención de obra.

Fuente: Autores a partir de AutoCAD.

instaló una estación meteorológica automática integrada Davis Vantage Pro $2^{\text {TM }}$ (Davis Instruments, Hayward (A) para la medición de las variables meteorológicas: velocidad y dirección del viento, temperatura, precipitación y radiación solar. El flujo de los monitores DustTrak fue calibrado antes del estudio usando un estándar de flujo acorde a recomendaciones del fabricante (TSI). Los equipos DustTrak se compararon con otros proyectos contramuestreos gravimétricos hechos con balanza de referencia de 5 cifras decimales, sensibles hasta $0.1 \mu \mathrm{g}$, empleando muestreadores personales con filtros de $37 \mathrm{~mm}$ para recoger simultáneamente muestras de particulado, observando excelentes resultados al comparar la masa muestreada por los DustTrak y la recolectada en los filtros. La masa reportada por los DustTrak está dentro del 15\% de la masa medida por el método gravimétrico. La pendiente de las calibraciones fue tendiente a uno, con línea de tendencia lineal y correlación superior o igual a 0.95. Así mismo se chequeó el cero de los DustTrak usando un filtro HEPA de acuerdo a especificaciones del fabricante y de igual manera le fueron cambiados los filtros internos del equipo. Los flujos y ceros de los aetalómetros fueron chequeados de la misma forma. Filtros frescos fueron usados cuando las trasmitancias en los aetalómetros así lo demandaron, siguiendo recomendaciones del fabricante. Las concentraciones de $\mathrm{PM}_{2.5^{\prime}}$ $\mathrm{PM}_{10}$ y $\mathrm{BC}$ menores a cero se corrigieron calculando el promedio de los valores absolutos de concentraciones menores o iguales al percentil 10 y agregándolo a cada dato de concentración registrado. Así mismo se realizaron cálculos de 
incertidumbre acorde a Orozco et al. [11] .

La vía arterial del barrio Caracolí, ubicada a 2.720 m sobre el nivel del mar, es un corredor comercial-habitacional multifamiliar, da acceso a varios equipamientos (colegios, jardines infantiles, entre otros), y a su vez conecta a Bogotá con el municipio de Soacha. Esta zona presenta un clima frío semiárido (Fsa) según modelo Climático Caldas-Lang, con precipitación promedio multianual (2002-2014) de 67 mms, humedad relativa media mensual multianual de $74 \%$, y rango de temperatura media multianual entre los $14,5-17^{\circ} \mathrm{C}$. Acorde a los datos suministrados por el IDEAM, presenta direcciones predominantes del viento hacia el sureste con un $27 \%$ y noroeste con un $17 \%$, con velocidades inferiores a $4 \mathrm{~m} / \mathrm{s}$ en su mayor parte. Las lluvias siguen un patrón bimodal, con precipitaciones concentradas en los periodos de marzo a comienzos de mayo, y desde, finales de septiembre a mediados de diciembre; y un periodo por debajo de la precipitación media de junio a agosto.

\subsection{Procesamiento de la información y eva- luación del impacto en calidad del aire}

Se utilizó la relación de $\mathrm{PM}_{2.5} / \mathrm{PM}_{10}$ para evaluar si su origen presentaba una asociación geológica (polvo fugitivo resuspendido) $(<0,4)$, por combustión $(>0,6)$ [15-17] o no definido, y la tendencia lineal entre $\mathrm{PM}_{2.5}$ y $\mathrm{BC}$, con un valor de $R^{2} \leq 0,6$, para descartar datos asociados a procesos de combustión.

Posteriormente, se valoró el impacto en calidad del aire, comparando los promedios aritméticos muestrales de concentración por fuente (geológica, por combustión y no definida), de los periodos del antes y después de la construcción vial completa. Conjuntamente se analizaron las concentraciones durante el re- emplazo de la superficie en afirmado, por una estructura de pavimento por parte de la UMV en el frente de obra.

\subsection{Procesamiento de la información y determinación de factores de emisión de polvo resuspendido}

Los criterios para la selección de datos a emplear para la estimación experimental de factores de emisión asociados a resuspensión fueron:

i. La relación de $\mathrm{PM}_{2.5} / \mathrm{PM}_{10}(<0,4)$ para asociar su origen a resuspensión de material particulado.

ii. La tendencia lineal entre $\mathrm{PM}_{2.5}$ y $\mathrm{PM}_{10^{\prime}}$ con un valor de $R^{2} \geq 0,9$, dentro de un conjunto al azar mínimo de cinco parejas de datos consecutivos, con lo que se podía inferir que se registra una misma fuente y comportamiento para $\mathrm{PM}_{2.5}$ y $\mathrm{PM}_{10}$.

iii. La tendencia lineal entre $\mathrm{PM}_{2.5}$ y $\mathrm{BC}$, con un valor de $\mathrm{R}^{2} \leq 0,6$, con el propósito de excluir las concentraciones de PM provenientes directamente de fuentes de combustión $\left(R^{2}>0,6\right)[11]$.

iv. Cada dato aceptado fue asociado a Vientos abajo (VAb) (VAb = NW, N, NE) o a Vientos Arriba (VAr) (VAr = SW, S, SE) del punto de monitoreo (Figura 2). Se promediaron los valores de concentración asociados a VAb y VAr para seguidamente obtener un valor delta de concentración $(\Delta C)(\Delta C=V A b-V A r)$.

Posteriormente, se emplearon dos modelos gaussianos de dispersión para determinar los factores de emisión, uno simple (SCREEN3) y otro refinado (AERMOD View). Se utilizaron modelos gaussianos dada su amplia aceptación por la comunidad científica y ser recomendados por agencias ambientales de diferentes 
países para ser usados con propósitos regulatorios [12].

AERMOD es un modelo de pluma, de estado estacionario, que asume que las concentraciones en todas las distancias están gobernadas por la meteorología promedio de una hora. Este modelo asume que bajo condiciones de estabilidad la distribución de concentraciones sigue una distribución normal tanto en la vertical como en la horizontal en un plano $y z$, en donde bajo una capa límite convectiva la distribución seguirá siendo gaussiana en el eje horizontal, pero se asumirá bigaussiana en el eje vertical [18].

La ecuación de difusión gaussiana relaciona los niveles de inmisión en un punto, con la cantidad de contaminantes vertidos a la atmósfera desde el foco emisor, teniendo en cuenta las condiciones de emisión del contaminante, y las características meteorológicas y topográficas del medio receptor [19]. La modelación refinada consiste en realizar una estimación mucho más precisa y exacta de la dispersión de contaminantes en un área determinada trayendo como resultado una mejor estimación del impacto generado por la fuente y proporcionando información de la efectividad de las medidas de control, estas características las posee el modelo AERMOD y es una de las razones por las cuales se consideró uno de los modelos adecuados [20].

Por otro lado, teniendo en cuenta que la concentración máxima resultante por modelo (AERMOD- SCREEN3) debía compararse con la concentración obtenida en los monitoreos Kerbside, de manera inversa se estimaron por prueba y error factores de emisión que generaran las concentraciones $(\Delta C)$ en el receptor específico (DustTrak) en las condiciones meteorológicas y geográficas presentes en la zona de estudio.
La fuente contaminante fue definida como una fuente de área donde el segmento de vía analizado posee una longitud de $210 \mathrm{~m}$ y una altura de 2,5 m acorde al establecimiento de la estación meteorológica. La altura de emisión asumida fue de $2 \mathrm{~m}$ acorde a la altura del monitor DustTrak. Las variables meteorológicas ingresadas en el preprocesador AERMET fueron temperatura $\left({ }^{\circ} \mathrm{C}\right)$, nubosidad (décimas), velocidad ( $\mathrm{m} / \mathrm{s})$, dirección del viento $\left({ }^{\circ}\right)$ y radiación solar $\left(\mathrm{W} / \mathrm{m}^{2}\right)$, estas variables a excepción de la nubosidad, fueron medidas en campo.

Para la corrida de AERMET, se utilizaron radiosondeos del aeropuerto El Dorado de la ciudad de Bogotá con el fin de obtener el perfil vertical de variables meteorológicas. En cuanto a los parámetros de superficie, se utilizaron los datos asociados al uso del suelo urbano para un periodo de un año (los valores de los parámetros de superficie se promediaron para las épocas del año), el albedo fue de 0,2075, Bowen Ratio 1,625, Surface Roughness 1. Dentro de la configuración inicial del modelo AERMOD se optó por la opción ADJ_U* la cual está diseñada para ajustar la velocidad de fricción de superficie, para el caso de bajas velocidades del viento y/o condiciones de estabilidad atmosférica. No se estableció el parámetro de building downwash, ya que la altura de las edificaciones ubicadas alrededor de la fuente en cuestión no está asociada a configuración de cañón urbano.

Posteriormente, AERMOD se configuró según las condiciones de la zona de estudio en un área de $1 \mathrm{~km}$ con centro en la fuente área de modelación. Se empleó una grilla de 441 receptores cartesianos y se ubicó como receptor discreto el equipo DustTrak teniendo en cuenta que las concentraciones se encontraran en el tiempo monitoreado. 
En el caso de SCREEN3, se ingresó la información de velocidad del viento y estabilidad atmosférica en clasificación de PasquillGifford.
Seguidamente, se realizó el cálculo del porcentaje de diferencia entre factores de emisión (FE) obtenidos y FE de diferentes investigaciones científicas.

$$
\text { Porcentaje de diferencia }=\frac{\text { (valor obtenido }- \text { valor de literatura) }}{\frac{\text { (valor obtenido }+ \text { valor de literatura) }}{2}} * 100 \%
$$

\section{RESULTADOS Y DISCUSIÓN}

\subsection{Evaluación del impacto en calidad del aire}

Al comparar las concentraciones promedio de PM del antes vs. después de la pavimentación se aprecian reducciones en fuente geológica, por combustión y no definida (Tabla 1). Las máximas concentraciones para ambas etapas de monitoreo se presentaron a las 12 pm \pm 2 horas, sin embargo únicamente antes de la pavimentación se realizaron aplicaciones rutinarias de agua a medio día por parte de sus habitantes en un intento por disminuir la suspensión de PM.

El material particulado asociado a fuente geológica (polvo fugitivo resuspendido) obtuvo el mayor porcentaje de reducción en concentración (Tabla 1), lo que apoyaría otras investigaciones en las que se han encontrado reducciones mayores al $90 \%$ en concentraciones de polvo fugitivo [7-8]. Este impacto positivo, traducido en menos efectos adversos al comercio, progreso en la calidad visual, aumento en la visibilidad y en sí en el mejoramiento en la calidad del aire es atribuible a la construcción de la vía (Figura 3).
Así mismo disminuyeron las concentraciones de PM asociado a fuente de combustión y a fuente no definida en más de un 37\%, influenciado por la disminución del tráfico estático que se presentaba por las malas condiciones de la vía, así como a la supresión de depósitos de polvo fugitivo una vez se realizó el proceso de pavimentación (Tabla 1). Se presentó de forma generalizada una disminución mayor en $\mathrm{PM}_{10}$ que en $\mathrm{PM}_{2.5}$ (Tabla 1), ya que el polvo fugitivo (aportante principal de partículas gruesas inhalables) se suprimió, no se presentaron embotellamientos y la perturbación del suelo desnudo que se presentaba antes de la pavimentación (por erosión antrópica y natural) disminuyó gracias a la intervención vial. Por ende, el porcentaje de número de datos de PM asociado a fuente geológica (polvo fugitivo resuspendido) y a fuente no definida (entre geológico y por combustión) disminuyó en 14 y 27,5 puntos porcentuales, respectivamente, mientras que el porcentaje de número de datos de PM asociado a fuente por combustión aumentó su representatividad en el número total de datos después de la pavimentación en 41,5 puntos porcentuales Tabla 1).

Acorde a los resultados de análisis durante la rehabilitación vial completa (Tabla 2), en la exca- 
Tabla 1. Resultados de análisis comparativos entre el antes y después de la pavimentación

\begin{tabular}{|c|c|c|c|c|c|c|c|c|c|}
\hline \multirow{2}{*}{$\begin{array}{l}\text { Clasificación } \\
\text { asociada }\end{array}$} & \multirow{2}{*}{ Estadístico } & \multicolumn{3}{|c|}{ Antes de pavimentar } & \multicolumn{3}{|c|}{ Después de pavimentar } & \multirow{2}{*}{$\begin{array}{c}\text { Cambio } \\
\text { en } \mathrm{PM}_{2.5} \\
(\%)\end{array}$} & \multirow{2}{*}{$\begin{array}{c}\text { Cambio } \\
\text { en } \mathrm{PM}_{10} \\
(\%)\end{array}$} \\
\hline & & $\begin{array}{l}\mathrm{PM}_{2.5} \\
\mu \mathrm{g} / \mathrm{m}^{3}\end{array}$ & $\begin{array}{c}\mathrm{PM}_{10} \\
\mu \mathrm{g} / \mathrm{m}^{3}\end{array}$ & $\begin{array}{c}\% \text { de } \\
\text { Datos }\end{array}$ & $\begin{array}{l}\mathrm{PM}_{2.5} \\
\mu \mathrm{g} / \mathrm{m}^{3}\end{array}$ & $\begin{array}{l}\mathrm{PM}_{10} \\
\mu \mathrm{g} / \mathrm{m}^{3}\end{array}$ & $\begin{array}{c}\% \text { de } \\
\text { Datos }\end{array}$ & & \\
\hline \multirow{3}{*}{ Fuente Geológica } & $\bar{x}$ & 381 & 1025 & \multirow{3}{*}{14,1} & 17 & 48 & \multirow{3}{*}{0,1} & \multirow{3}{*}{$-95,7$} & \multirow{3}{*}{$-95,4$} \\
\hline & $\sigma$ & 313 & 860 & & 13 & 37 & & & \\
\hline & Rango & $\begin{array}{l}134- \\
1000\end{array}$ & $\begin{array}{l}364- \\
2732\end{array}$ & & $\begin{array}{l}1- \\
32\end{array}$ & $\begin{array}{l}5- \\
92\end{array}$ & & & \\
\hline \multirow{3}{*}{$\begin{array}{l}\text { Fuente por } \\
\text { combustión }\end{array}$} & $\bar{x}$ & 87 & 117 & \multirow{3}{*}{51,5} & 54 & 70 & \multirow{3}{*}{93} & \multirow{3}{*}{$-37,4$} & \multirow{3}{*}{$-40,3$} \\
\hline & $\sigma$ & 29 & 41 & & 23 & 25 & & & \\
\hline & Rango & $\begin{array}{l}57- \\
126\end{array}$ & $\begin{array}{l}72- \\
170\end{array}$ & & $\begin{array}{l}32- \\
90\end{array}$ & $\begin{array}{l}44- \\
104\end{array}$ & & & \\
\hline \multirow{3}{*}{ Fuente no definida } & $\bar{x}$ & 160 & 339 & \multirow{3}{*}{34,4} & 86 & 161 & \multirow{3}{*}{6,9} & \multirow{3}{*}{$-46,3$} & \multirow{3}{*}{$-52,5$} \\
\hline & $\sigma$ & 75 & 157 & & 82 & 162 & & & \\
\hline & Rango & $\begin{array}{l}87- \\
288\end{array}$ & $\begin{array}{l}176- \\
594\end{array}$ & & $\begin{array}{l}29- \\
219\end{array}$ & $\begin{array}{l}52- \\
431\end{array}$ & & & \\
\hline
\end{tabular}

Nota: Se analizaron en total 12.786 datos antes de pavimentar y 15.070 datos después de pavimentar. Cálculos con base en promedio diario. $(\bar{X})$ promedio aritmético. $(\sigma)$ desviación estándar. (Rango) dato mínimo - dato máximo. Cambio en porcentaje de concentración de contaminante con base en el valor del promedio aritmético por fuente.

Fuente: Elaboración propia.

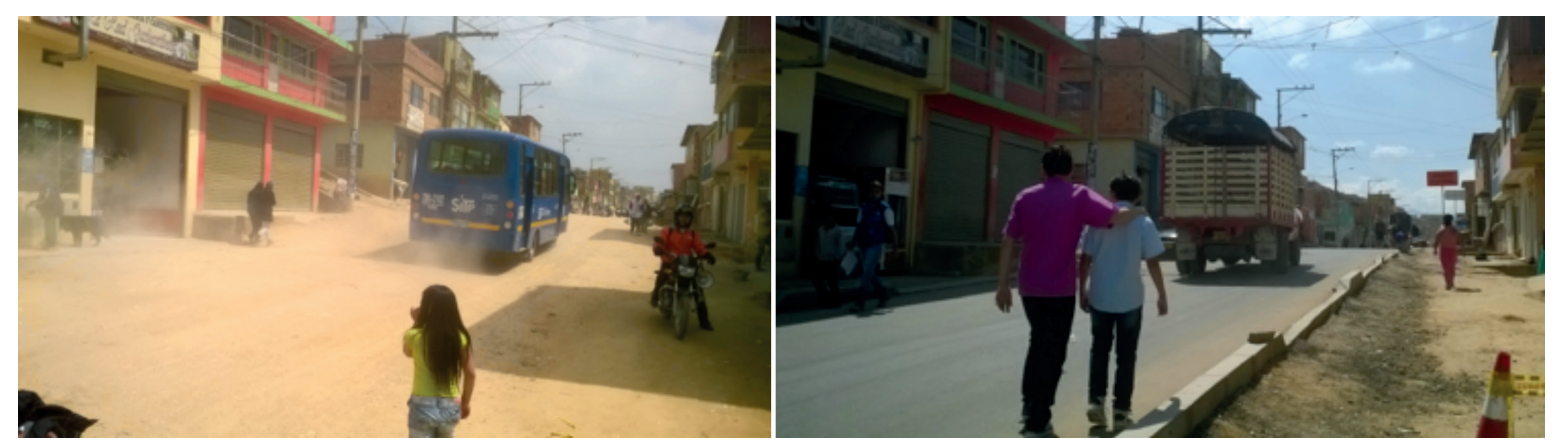

Figura 3. Antes vs. Después de la pavimentación.

Fuente: Elaboración propia. 
vación por métodos mecánicos frente al punto de monitoreo se obtuvieron los máximos valores promedios para $\mathrm{PM}_{2.5}$ y $\mathrm{PM}_{10}$ asociados a fuente geológica, dado su aporte a la generación y trituración de detritos geológicos. Por su parte, el PM asociado a fuente por combustión tuvo su mayor presencia (No. datos/día) cuando se colocó la carpeta asfáltica (23/04/2015) con un $98,3 \%$ del total de datos, pero la mayor concentración por día de esta etapa se puede asociar posiblemente a la maquinaria de excavación que estuvo al frente del punto de monitoreo la mayor parte del tiempo y al cuello de botella vehicular que se presentó. Después de la aplicación del fresado estabilizado (durante la pavimentación) las concentraciones tendieron a tener valores por debajo de los registrados en la excavación, ya que el fresado estabilizado posee una superficie pegajosa y se presenta como conglomerado, haciendo más difícil su resus-

Tabla 2. Resultados de análisis durante los días más representativos del proceso de pavimentación

\begin{tabular}{|c|c|c|c|c|c|c|c|c|c|c|c|c|}
\hline \multirow{3}{*}{ Clasificación } & \multicolumn{3}{|c|}{ 13/01/2015 } & \multicolumn{3}{|c|}{$14 / 01 / 2015$} & \multicolumn{3}{|c|}{$15 / 01 / 2015$} & \multicolumn{3}{|c|}{ 16/01/2015 } \\
\hline & \multicolumn{3}{|c|}{$\begin{array}{l}\text { Excavación frente al } \\
\text { punto de monitoreo }\end{array}$} & \multicolumn{3}{|c|}{$\begin{array}{l}\text { Aplicación de fresado estabiliza- } \\
\text { do frente al punto de monitoreo }\end{array}$} & \multicolumn{3}{|c|}{$\begin{array}{l}\text { Excavación y aplicación de } \\
\text { fresado (carril más cercano) }\end{array}$} & \multicolumn{3}{|c|}{$\begin{array}{l}\text { Excavación y aplicación de } \\
\text { fresado (carril más cercano) }\end{array}$} \\
\hline & $\begin{array}{l}\mathrm{PM}_{2.5} \\
\mu \mathrm{g} / \mathrm{m}^{3}\end{array}$ & $\begin{array}{l}\mathrm{PM}_{10} \\
\mu \mathrm{g} / \mathrm{m}^{3}\end{array}$ & $\mathrm{n}$ & $\begin{array}{l}\mathrm{PM}_{2.5} \\
\mu \mathrm{g} / \mathrm{m}^{3}\end{array}$ & $\begin{array}{l}\mathrm{PM}_{10} \\
\mu \mathrm{g} / \mathrm{m}^{3}\end{array}$ & $\mathrm{n}$ & $\begin{array}{l}\mathrm{PM}_{2.5} \\
\mu \mathrm{g} / \mathrm{m}^{3}\end{array}$ & $\begin{array}{l}\mathrm{PM}_{10} \\
\mu \mathrm{g} / \mathrm{m}^{3}\end{array}$ & $\mathrm{n}$ & $\begin{array}{l}\mathrm{PM}_{2.5} \\
\mu \mathrm{g} / \mathrm{m}^{3}\end{array}$ & $\begin{array}{l}\mathrm{PM}_{10} \\
\mu \mathrm{g} / \mathrm{m}^{3}\end{array}$ & n \\
\hline Fuente Geológica* & 233 & 627 & 575 & 147 & 394 & 898 & 107 & 285 & 77 & 164 & 437 & 345 \\
\hline $\begin{array}{l}\text { Fuente por } \\
\text { combustión* }\end{array}$ & 234 & 360 & 68 & 174 & 240 & 146 & 84 & 120 & 1473 & 102 & 149 & 526 \\
\hline \multirow[t]{2}{*}{ Fuente no definida* } & 192 & 424 & 1681 & 122 & 270 & 1354 & 88 & 178 & 974 & 124 & 268 & 1585 \\
\hline & \multicolumn{2}{|c|}{ TOTAL } & 2324 & \multicolumn{2}{|c|}{ TOTAL } & 2398 & \multicolumn{2}{|c|}{ TOTAL } & 2524 & \multicolumn{2}{|c|}{ TOTAL } & 2456 \\
\hline \multirow{3}{*}{ Clasificación } & \multicolumn{3}{|c|}{$17 / 01 / 2015$} & \multicolumn{3}{|c|}{$21 / 01 / 2015$} & \multicolumn{3}{|c|}{$22 / 04 / 2015$} & \multicolumn{3}{|c|}{ 23/04/2015 } \\
\hline & \multicolumn{3}{|c|}{$\begin{array}{c}\text { Excavación y aplicación } \\
\text { de fresado (carril más } \\
\text { cercano) }\end{array}$} & \multicolumn{3}{|c|}{$\begin{array}{l}\text { Excavación cercana al punto de } \\
\text { monitoreo (carril más lejano) }\end{array}$} & \multicolumn{3}{|c|}{ Aplicación de emulsión } & \multicolumn{3}{|c|}{$\begin{array}{l}\text { Aplicación de mezcla } \\
\text { asfáltica densa en caliente }\end{array}$} \\
\hline & $\begin{array}{l}\mathrm{PM}_{2.5} \\
\mu \mathrm{g} / \mathrm{m}^{3}\end{array}$ & $\begin{array}{l}\mathrm{PM}_{10} \\
\mu \mathrm{g} / \mathrm{m}^{3}\end{array}$ & n & $\begin{array}{l}\mathrm{PM}_{2.5} \\
\mu \mathrm{g} / \mathrm{m}^{3}\end{array}$ & $\begin{array}{l}\mathrm{PM}_{10} \\
\mu \mathrm{g} / \mathrm{m}^{3}\end{array}$ & $\mathrm{n}$ & $\begin{array}{l}\mathrm{PM}_{2.5} \\
\mu \mathrm{g} / \mathrm{m}^{3}\end{array}$ & $\begin{array}{l}\mathrm{PM}_{10} \\
\mu \mathrm{g} / \mathrm{m}^{3}\end{array}$ & $\mathrm{n}$ & $\begin{array}{l}\mathrm{PM}_{2.5} \\
\mu \mathrm{g} / \mathrm{m}^{3}\end{array}$ & $\begin{array}{l}\mathrm{PM}_{10} \\
\mu \mathrm{g} / \mathrm{m}^{3}\end{array}$ & $n$ \\
\hline Fuente Geológica* & - & - & 0 & 38 & 105 & 806 & - & - & 0 & - & - & 0 \\
\hline $\begin{array}{l}\text { Fuente por } \\
\text { combustión* }\end{array}$ & 105 & 124 & 2462 & 72 & 89 & 544 & 26 & 35 & 2127 & 71 & 85 & 2586 \\
\hline \multirow[t]{2}{*}{ Fuente no definida* } & 179 & 343 & 65 & 37 & 83 & 1158 & 63 & 111 & 40 & 76 & 129 & 45 \\
\hline & \multicolumn{2}{|c|}{ TOTAL } & 2527 & \multicolumn{2}{|c|}{ TOTAL } & 2508 & \multicolumn{2}{|c|}{ TOTAL } & 2167 & \multicolumn{2}{|c|}{ TOTAL } & 2631 \\
\hline
\end{tabular}

Nota: *Calculado del promedio aritmético. (n) Número de datos

Fuente: Elaboración propia. 
pensión (Tabla 2). Asi mismo, las concentraciones de PM asociadas a resuspensión del durante la pavimentación disminuyeron con respecto al antes de la pavimentación en parte por las medidas de control de emisiones atmosféricas por parte de la UMV (tela para cerramiento y riego de agua) y a la habilitación del paso vehicular por un solo carril.

\subsection{Determinación de factores de emisión de polvo resuspendido}

En la Tabla 3 se muestra un resumen de los datos resultantes de $\mathrm{FE}$ calculados para $\mathrm{PM}_{10}$ y $\mathrm{PM}_{2.5}$ en las etapas del antes, durante y después de la pavimentación.

Tabla 3. Concentraciones de material particulado y factores de emisión (FE) obtenidos para las 3 etapas a partir de modelos de dispersión

\begin{tabular}{|c|c|c|c|c|}
\hline ETAPA & VARIABLES & $\mathbf{P M}_{10}$ & $\mathrm{PM}_{2.5}$ & $\begin{array}{l}\text { ESTABILIDAD } \\
\text { ATMOSFÉRICA }\end{array}$ \\
\hline \multirow{6}{*}{$\begin{array}{c}(1) \\
\text { ANTES } \\
(n=13132)\end{array}$} & Datos asociados a resuspensión de PM (\%)* & 30,7 & 30,7 & \multirow{6}{*}{$\begin{array}{c}\text { (B) } \\
\text { Atmósfera } \\
\text { moderadamente } \\
\text { inestable } \\
\text { Rad. Solar }=35,6 \mathrm{cal} / \\
\mathrm{cm}^{2 * h}\end{array}$} \\
\hline & Promedio VAb $\left(\mu \mathrm{g} / \mathrm{m}^{3}\right)$ & 381,5 & 195,70 & \\
\hline & Promedio $\operatorname{VAr}\left(\mu \mathrm{g} / \mathrm{m}^{3}\right)$ & 302,0 & 190,2 & \\
\hline & Delta $\left(\mu \mathrm{g} / \mathrm{m}^{3}\right)$ & 79,5 & 5,5 & \\
\hline & FEAERMOD $\left(\mu \mathrm{g} / \mathrm{m}^{2 *} \mathrm{~s}\right)$ & $60 \pm 0,50$ & $4,3 \pm 0,20$ & \\
\hline & FE SCREEN3 $\left(\mu \mathrm{g} / \mathrm{m}^{2 *} \mathrm{~s}\right)$ & $25 \pm 0,50$ & $1,7 \pm 0,20$ & \\
\hline \multirow{6}{*}{$\begin{array}{c}(2) \\
\text { DURANTE } \\
(\mathrm{n}=12480)\end{array}$} & Datos asociados a resuspensión de PM (\%)* & 4,7 & 4,7 & \multirow{6}{*}{$\begin{array}{c}(A-B) \\
\text { atmósfera } \\
\text { extremadamente } \\
\text { inestable } \\
\text { a moderadamente } \\
\text { inestable } \\
\text { Rad. Solar }=41,3 \mathrm{cal} / \\
\mathrm{cm}^{2 * h}\end{array}$} \\
\hline & Promedio VAb $\left(\mu \mathrm{g} / \mathrm{m}^{3}\right)$ & 257,0 & 124,51 & \\
\hline & Promedio $\operatorname{VAr}\left(\mu \mathrm{g} / \mathrm{m}^{3}\right)$ & 214,5 & 107,7 & \\
\hline & Delta $\left(\mu \mathrm{g} / \mathrm{m}^{3}\right)$ & 42,5 & 16,8 & \\
\hline & FEAERMOD $\left(\mu \mathrm{g} / \mathrm{m}^{2 *} \mathrm{~s}\right)$ & $28 \pm 0,27$ & $11 \pm 0,13$ & \\
\hline & FE SCREEN3 $\left(\mu \mathrm{g} / \mathrm{m}^{2 *} \mathrm{~s}\right)$ & $13,0 \pm 0,27$ & $3,6 \pm 0,13$ & \\
\hline \multirow{6}{*}{$\begin{array}{c}\text { (3) } \\
\text { DESPUÉS } \\
(\mathrm{n}=15071)\end{array}$} & Datos asociados a resuspensión de PM (\%)* & 0,1 & 0,1 & \multirow{6}{*}{$\begin{array}{c}\text { (A) } \\
\text { atmósfera } \\
\text { extremadamente } \\
\text { inestable } \\
\text { Rad. Solar }=53,1 \mathrm{cal} / \\
\mathrm{cm}^{2} * h\end{array}$} \\
\hline & Promedio VAb $\left(\mu \mathrm{g} / \mathrm{m}^{3}\right)$ & 79,4 & 62,11 & \\
\hline & Promedio $\operatorname{VAr}\left(\mu \mathrm{g} / \mathrm{m}^{3}\right)$ & 75,0 & 55,2 & \\
\hline & Delta $\left(\mu \mathrm{g} / \mathrm{m}^{3}\right)$ & 4,4 & 6,9 & \\
\hline & FE AERMOD $\left(\mu \mathrm{g} / \mathrm{m}^{2 *} \mathrm{~s}\right)$ & $0,2 \pm 0,10$ & $3,0 \pm 0,08$ & \\
\hline & FE SCREEN3 $\left(\mu \mathrm{g} / \mathrm{m}^{2 *} \mathrm{~s}\right)$ & $0,8 \pm 0,10$ & $1,2 \pm 0,08$ & \\
\hline
\end{tabular}

* Porcentaje de datos que cumplen con los cuatro criterios establecidos en el numeral 1.3 de la metodología. ** Estabilidad definida según Pasquill-Girfford.

Fuente: Elaboración propia. 
En la determinación de FE los eventos de contaminación antes y durante la pavimentación, estuvieron asociados a un número de datos superior a 10.000. En la etapa 3 (después de la pavimentación) aunque se presentó un número de datos similar, solo el $0,1 \%$ de los datos estuvo asociado a concentraciones de material particulado coligado a resuspensión, así mismo las concentraciones asociadas a vientos arriba (concentraciones de fondo o Background) fueron mayores que las concentraciones asociadas a vientos abajo (Background + Fuente), lo que implicaría que la metodología no se ajusta a un bajo número de datos de concentración asociados a resuspensión de material particulado de la fuente en cuestión, en este caso la vía pavimentada.

Por otro lado, al comparar espacialmente la dispersión del contaminante (antes vs. después de la pavimentación) (Figuras 4a y 4b) (Figura 5), en ambos modelos se observó una reducción homogénea de la concentración, a medida que aumenta la distancia del receptor (DustTrak) ubicado en la vía principal de Caracolí, asociado a procesos de transporte por di-

a)

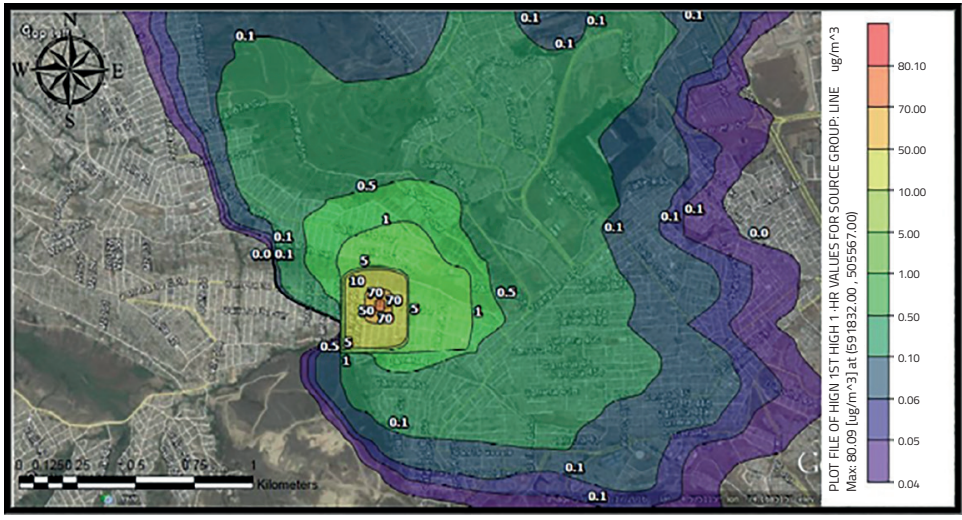

b)

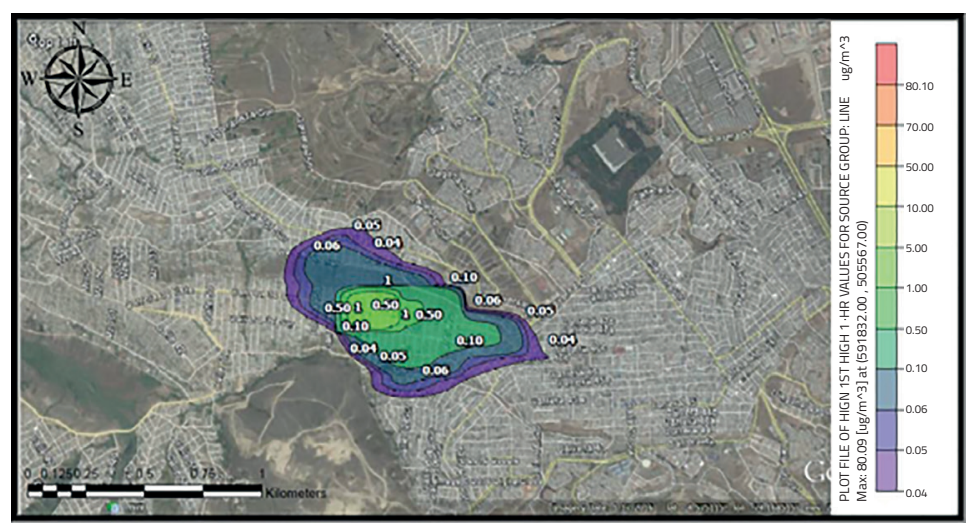

Figura 4. Isolíneas de concentración de PM 10 asociado a resuspensión en la vía principal del Barrio Caracolí, Localidad de Ciudad Bolívar a. Vía sin pavimentar b. Vía pavimentada.

Fuente: Elaboración propia a partir de AERMOD. 


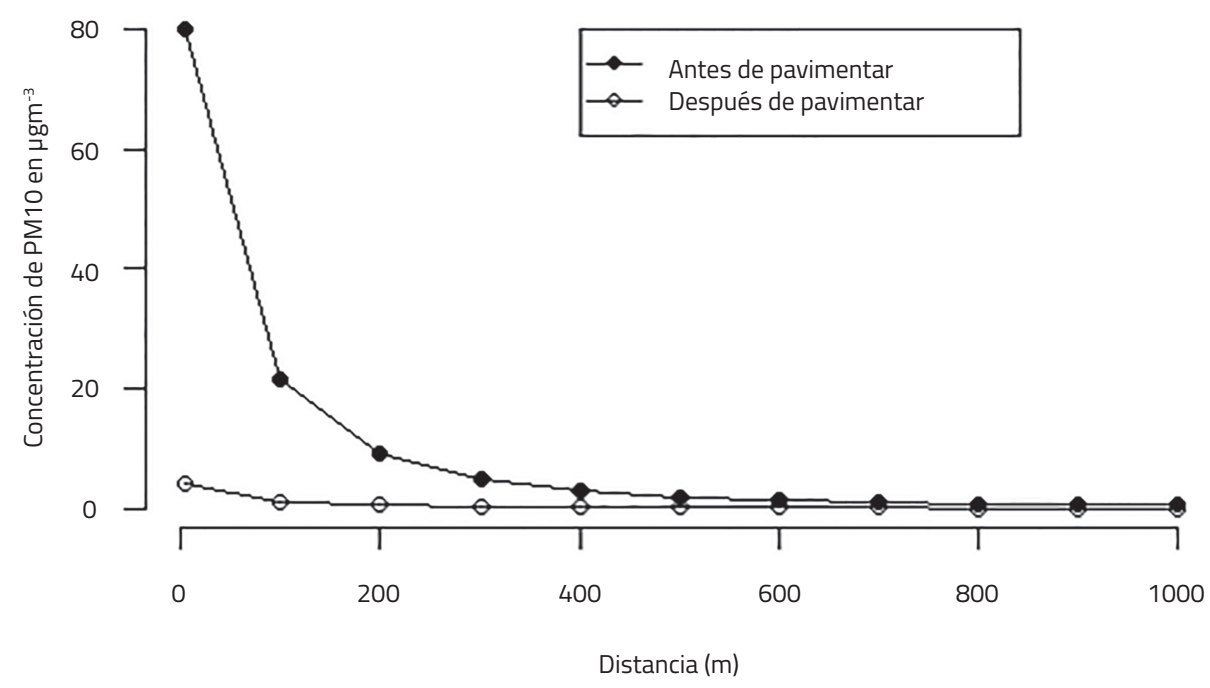

Figura 5. Perfil de concentración de PM 10 asociado a resuspensión en la vía principal del Barrio Caracolí, Localidad de Ciudad Bolivar

Fuente: Elaboración propia a partir de datos de modelación en SCREEN3.

fusión y advección del material particulado. Se observa una reducción del área de influencia indirecta (radio de $1 \mathrm{~km}$ ) de los contaminantes, así como de los valores de concentración espacial del PM, después de la pavimentación.

La diferencia entre los FE comparados (Tabla 4) puede asociarse al factor de actividad de la zona, puesto que zonas con mayor tráfico vehicular acarrean mayores emisiones. Por otra parte se puede observar que la diferencia entre los FE entre modelos es de $7,4 \mu \mathrm{g} / \mathrm{m}^{2 *} \mathrm{~s}$ para $\mathrm{PM}_{2.5}$ y $15 \mu \mathrm{g} / \mathrm{m}^{2 *} \mathrm{~s}$ para $\mathrm{PM}_{10}$ (Tabla 4). Al comparar el factor de emisión de $\mathrm{PM}_{2.5}$ obtenido con el modelo AERMOD para la etapa 2 (durante la pavimentación) frente a lo obtenido por Orozco et al. [11], y los FE obtenidos para la Comisión Nacional del Medio Ambiente Región Metropolitana (CONAMA) [22] en la ciudad de
Santiago de Chile, las diferencias entre los FE de la literatura y de esta investigación se asocian a las condiciones de cada estudio como es el caso del tipo de mantenimiento vial, así como a las condiciones geográficas y climatológicas específicas de la zona.

Para la obtención de factores de emisión en unidades de (g/vkt) se utilizó el promedio de vehículos que transitaron en el segmento analizado de la vía principal del barrio Caracolí, por etapa y tiempo de monitoreo. Se obtuvo un promedio de 837 veh/día antes de la pavimentación de tipo moto, automóvil, campero y camionetas, buseta y busetón, camión de 2 ejes pequeño y grande, microbús y en menor medida tipo camión de 3 y 4 ejes, bus largo y taxi. Después de la pavimentación se obtuvieron 835 veh/día del mismo tipo. 
En el caso de los FE de una vía sin pavimentar (etapa 1) la comparación (Tabla 5) muestra que esta investigación presenta valores cercanos a los que se obtuvieron en EUA y la frontera EUA-México y alejados con los conseguidos en Spokane Washington (Tabla 6). En el caso de vía pavimentada se presentan valores más cerca- nos entre estudios locales. Como era esperable tanto en la investigación como en la literatura, la vía pavimentada presenta valores más bajos con respecto a los presentados en vía sin pavimentar. La relación de $\left[\mathrm{PM}_{10}>\mathrm{PM}_{2.5}\right]$ no se obtuvo en vía pavimentada por parte de los autores, asociado a que la metodología propues-

Tabla 4. Comparación de factores de emisión durante proceso de pavimentación

\begin{tabular}{|c|c|c|c|c|}
\hline \multirow{2}{*}{ ETAPA } & FUENTE & ZONA & $\mathbf{P M}_{\mathbf{1 0}} \mathbf{\mu g} / \mathbf{m}^{\mathbf{2 *}} \mathbf{S}$ & $\mathbf{P M}_{\mathbf{2 . 5}} \mathbf{\mu g} / \mathbf{m}^{\mathbf{2 *}} \mathbf{s}$ \\
\hline \multirow{4}{*}{$\begin{array}{c}\text { Durante la } \\
\text { Pavimentación }\end{array}$} & FE AERMOD & Bogotá & $28 \pm 0,27$ & $11 \pm 0,13$ \\
\cline { 2 - 5 } & Orozco et al [11] & Bogotá & 191,7 & 14,5 \\
\cline { 2 - 5 } & EPA [21] & EUA & 101,0 & - \\
\cline { 2 - 5 } & FE SCREEN3 & Bogotá & $13,0 \pm 0,27$ & $3,6 \pm 0,13$ \\
\cline { 2 - 5 } & CONAMA [22] & $\begin{array}{c}\text { Santiago de } \\
\text { Chile }\end{array}$ & 4,3 & 2,5 \\
\hline
\end{tabular}

Fuente: Elaboración propia.

Tabla 5. Comparación en FE para vía sin pavimentar (antes) y vía pavimentada (después)

\begin{tabular}{|c|c|c|c|c|}
\hline ETAPAS & FACTORES DE EMISION & ZONA & $\mathrm{PM}_{10}$ g/vkt & $\mathrm{PM}_{2.5} \mathrm{~g} / \mathrm{vkt}$ \\
\hline \multirow{4}{*}{$\begin{array}{l}\text { Antes de } \\
\text { Pavimentar }\end{array}$} & FE AERMOD & Bogotá & 7,8 & 0,6 \\
\hline & EPA [17] & EUA & 6,4 & 1,1 \\
\hline & Kavouras et al [23] & $\begin{array}{c}\text { Frontera } \\
\text { EUA- México }\end{array}$ & 5.0 & - \\
\hline & Claiborn et al [24] & Spokane Washington & 5.8 & - \\
\hline \multirow{5}{*}{$\begin{array}{l}\text { Después de } \\
\text { Pavimentar }\end{array}$} & FE AERMOD & Bogotá & 0.30 & 0,4 \\
\hline & Venkatram et al [25] & Riverside California & 0.20 & - \\
\hline & Etyemezian et al [26] & Treasure Valley & 0.56 & - \\
\hline & Claiborn et al [24] & Spokane Washington & 0.50 & - \\
\hline & EPA [25] & EUA & 0.64 & - \\
\hline
\end{tabular}

Fuente: Elaboración propia. 
ta no es aplicable a porcentaje de datos de PM asociados a resuspensión del 0,1\%.

Estos porcentajes de diferencia (Tabla 6) se asocian a elementos diferenciales entre los estudios tales como mayor tráfico vehicular, tipos de vehículos que transitan por el área de estudio, velocidad promedio de los vehículos, uso industrial del suelo, características topográficas del área en cuestión, variables meteorológicas del área de estudio y las condiciones de monitoreo. En el caso de las diferencias encontradas para la etapa del durante la pavimentación, se evidencian características no homogéneas como ubicación de la tela para cerramiento, el avance de las obras en el segmento, el cambio en la ubicación de los equipos asociado a la protección de los mismos, entre otros aspectos.

Las diferencias de los factores de emisión entre los segmentos de caminos no pavimenta- dos evaluados en la investigación de Kavouras et al., donde se utilizó la técnica conocida como TRAKER [23], permitieron demostrar que estas eran estadísticamente significativas, indicando que había una variación espacial fuerte de emisiones de caminos no pavimentados, reflejadas en características diferentes como el tipo de suelo de la zona a evaluar, y condiciones del tráfico (la frecuencia, el tipo de vehículo, velocidad), para lo cual se denota que es necesario una obtención de factores de emisión de tipo local.

Adicionalmente según Venkatram et al. [25] el modelo del AP-42 es una relación netamente estadística entre estimaciones de emisión y el contenido de sedimentos de la vía, que carece de contenido mecánico (cuantificación de polvo resuspendido por paso vehicular), por lo que no puede ser usado en series de datos que no se basen

Tabla 6. Diferencia porcentual entre FE para vía sin pavimentar (antes) y vía pavimentada (después)

\begin{tabular}{|c|c|c|c|}
\hline ETAPA & COMPARATIVO & $\begin{array}{l}\text { DIFERENCIA EN } \\
\text { PM }_{10}(\%)\end{array}$ & $\begin{array}{c}\text { DIFERENCIA EN PM } \\
(\%)\end{array}$ \\
\hline \multirow{3}{*}{$\begin{array}{l}\text { Antes de } \\
\text { pavimentar }\end{array}$} & FE AERMOD vs. EPA [17] & 19 & 58 \\
\hline & FE AERMOD vs. Claiborn et al [24] & 30 & - \\
\hline & FE AERMOD vs. Kavouras [23] & 44 & \\
\hline \multirow{4}{*}{$\begin{array}{l}\text { Después de } \\
\text { pavimentar }\end{array}$} & FE AERMOD vs. Venkatram et al [25] & 26 & - \\
\hline & FE AERMOD vs. Claiborn et al [24] & 63 & - \\
\hline & FE AERMOD vs. Etyemezian et al [26] & 73 & \\
\hline & FE AERMOD vs. EPA [25] & 84 & - \\
\hline
\end{tabular}

Fuente: Elaboración propia. 
esencialmente en la deducción del contenido de sedimentos. Asimismo, las emisiones de $\mathrm{PM}_{10}$ estimadas mediante el uso de la AP-42 pueden desviarse por encima del orden de magnitud, por lo que se necesitan métodos más confiables para determinar los factores de emisión de vías pavimentadas y sin pavimentar, como es el uso de modelos de dispersión que representen con precisión los comportamientos de la zona de estudio.

El uso de la diferencia entre las concentraciones vientos arriba y vientos abajo de la fuente, para inferir el balance de masa contaminante de la vía pavimentada usada tanto en la AP-42 [25] como en esta investigación, es correcta siempre y cuando esta información sea considerada para cada área de estudio en particular y sea o esté acompañada de otras técnicas complementarias a las de cálculo de sedimento.

\section{CONCLUSIONES}

Este estudio determinó factores de emisión de material resuspendido antes, durante y después de la pavimentación de una vía en afirmado en la ciudad de Bogotá. Estos factores son útiles en la generación de inventarios de emisiones de polvo resuspendido en vías pavimentadas, no pavimentadas y en vías en proceso de pavimentación. Para la estimación de los FE, se aplicó una metodología novedosa de medición de concentraciones ambientales y modelación inversa.

La pavimentación parcial de la vía arterial en afirmado de la localidad de Ciudad Bolívar por parte de la UMV impactó positivamente en la calidad del aire, reduciendo las concentraciones de $\mathrm{PM}_{2.5}$ y $\mathrm{PM}_{10}$ asociadas a resuspensión en un $95 \%$. No obstante, disminuyeron las concentraciones de $\mathrm{PM}_{2.5}$ y $\mathrm{PM}_{10}$ asociadas a fuente por combustión y a fuente no definida en más de un $37 \%$.

Las concentraciones más altas de material particulado en la pavimentación se presentaron durante la excavación y tendieron a disminuir después de la aplicación del fresado estabilizado. El material particulado se asoció principalmente con fuente por combustión en la etapa de aplicación de mezcla asfáltica densa en caliente.

Los factores de emisión obtenidos para las etapas 1 (antes de la pavimentación) y 3 (después de la pavimentación), al ser comparados con los generados por la EPA, tienen un porcentaje de diferencia del $19 \%$, y $26 \%$ respectivamente y se obtuvo un porcentaje de diferencia del $58 \%$ para FE asociado a $\mathrm{PM}_{2.5}$ (etapa 1). En el caso de otras investigaciones donde se utilizan diferentes metodologías para la obtención de factores de emisión para una vía sin pavimentar, se obtuvo que los porcentajes de diferencia oscilan entre el $30 \%$ al $44 \%$ y para una vía pavimentada los porcentajes de diferencia oscilan entre el $63 \%$ al $84 \%$. Lo anterior permite mencionar que los factores de emisión deberían estimarse para condiciones geográficas, meteorológicas y de actividad específicas.

\section{AGRADECIMIENTOS}

Los autores agradecen a la Unidad de Mantenimiento Vial (UMV) del Distrito por el apoyo en el desplazamiento de los investigadores hasta la zona de Ciudad Bolívar, donde se realizaron los procesos de pavimentación. Además, un especial agradecimiento a la Universidad de La Salle por la financiación de la presente investigación. 


\section{REFERENCIAS}

[1] Organización Mundial de la Salud. (2014). 7 millones de muertes cada año debidas a la contaminación atmosférica. En: http:// www.who.int/mediacentre/news/releases/2014/air-pollution/es/ (30 septiembre del 2015).

[2] Rodríguez, N., Martínez, V., Sarmiento, R., Medina, K., \& Hernández, L. (2013). Factores de riesgo para enfermedad respiratoria en población de 5 a 14 años de una Localidad de Bogotá, 2012-2013. Rev. Salud Pública, 15 (3), pp. 408-420.

[3] Pachón, J. (2013). Tendencias en investigación sobre calidad del aire en Colombia a partir de los resultados del IV Congreso Colombiano y Conferencia Internacional de Calidad del Aire y Salud Pública (Casap) 2013. Épsilon, 21 (2), pp. 13-40.

[4] Beltrán, D., Belalcázar, L. C., \& Rojas, N. (2012). Emisiones vehiculares de material particulado (PM2.5 y PM10) por resuspensión de polvo y abrasión en Bogotá. Asociación Colombiana de Ingeniería Sanitaria y Ambiental, 231, pp. 25-32.

[5] O'Ryan, R. Larraguibel, L (2000). Contaminación del aire en Santiago: ¿qué es, qué se ha hecho, qué falta? Revista Perspectivas (Departamento de Ingeniería Industrial, Universidad de Chile), 4 (1), pp. 153-191.

[6] Universidad de Santiago de Chile. (2014). Estudio "Actualización y sistematización del inventario de emisiones de contaminantes atmosféricos en la Región Metropolitana". En: http://www.sinia.cl/1292/articles-56914_Inf_Inventarios_FINAL.pdf (30 septiembre del 2015).

[7] Succarieh, M. (1992). Control of Dust Emissions from unpaved roads. Fairbanks, Alaska 99775: Alaska Cooperative Trans- portation and Public Facilities Research Program, $55 \mathrm{p}$.

[8] Watson, J. G., \& Chow, J. C. (2000). Reconciling Urban Fugitive Dust Emissions Inventory and Ambient Source Contribution Estimates: Summary of Current Knowledge and Needed Research. DRI Document No 6110.4F. Nevada, USA: Desert Research Institute. Energy and Environmental Engineering Center, $240 \mathrm{p}$.

[9] The World Bank. (2011). Quantifying the impacts of vehicle-generated dust: a comprehensive approach. Washington DC 20433: The International Bank for Reconstruction and Development / The World Bank, $71 \mathrm{p}$.

[10] Alaska Department of Environmental Conservation. (2015). Top Ten Dust Control Techniques. En: https://dec.alaska.gov/air/ anpms/Dust/topten_dustctrl2.htm (30 septiembre del 2015)

[11] Orozco, K. Huelvas, A. Galvis, B. (2015). Determinación de factores de emisión para PM10 proveniente de construcción de vías, edificaciones y canteras en Bogotá (Trabajo de Grado). Facultad de Ingeniería, Universidad de la Salle, Bogotá, 100 p.

[12] Instituto Tecnológico y de Estudios Superiores de Monterrey. (2008). Guía nacional de modelación de calidad del aire: Convenio de Cooperación Financiera, Científica y Tecnológica 049 del 2008. Toluca, México: Centro de Investigación en Mecatrónica Automotriz - CIMA, $114 \mathrm{p}$.

[13] Ministerio de Ambiente y Desarrollo Sostenible (MADS). (2008). Protocolo para el monitoreo y seguimiento de la calidad del aire. Bogotá: Ministerio de Ambiente y Desarrollo Sostenible, $287 \mathrm{p}$.

[14] United States Environmental Protection Agency (EPA). (1999). Compendium of Methods for the Determination of Inorganic Compounds in Ambient Air. Washington, DC: EPA, $21 \mathrm{p}$. 
[15] Querol, X., Alastuey, A., Ruiz, C. R., Artiñano, B., Hansson, H. C., Harrison, R. M., Schneider, J. (2004). Speciation and origin of PM10 and PM2.5 in selected European cities. Atmospheric Environment, 38 (38), pp. 6547-6555. doi: 10.1016/j. atmosenv.2004.08.037

[16] Galvis, B. \& Rojas, N. (2006). Relación entre PM2.5 y PM10 en la Ciudad de Bogotá. ACTA NOVA, 3 (2), pp. 336-353.

[17] United States Environmental Protection Agency (EPA). (2006). Background Document for Revisions to Fine Fraction Ratios Used for AP-42 Fugitive Dust Emission Factor. En: http://www.epa.gov/ttn/chief/ ap42/ch13/bgdocs/b13s02.pdf (30 septiembre del 2015).

[18] United States Environmental Protection Agency (EPA). (2004). AERMOD: Description of model formulation. Washington, DC: EPA, $91 \mathrm{p}$.

[19] Manzur, M. E., Benzal, G., \& Gonzales, S. N. (2012). Modelo de Dispersión de Contaminantes Atmosféricos. Buenos Aires.

[20] United States Environmental Protection Agency (EPA). (2005). Revision to the guideline on air quality models: adoption of a preferred general purpose (flat and complex terrain) Dispersion model and other revisions; Final rule. Washington,DC: EPA, 45 p.

[21] United States Environmental Protection Agency (EPA). (1995). AP-42, Section 13.2.4 Aggregate handling and storage piles. Washington,DC: EPA.

[22] Comisión Nacional del Medio Ambiente Región Metropolitana (CONAMA RM).
(2007). Actualización del Inventario de Emisiones de Contaminantes Atmosféricos en la Región Metropolitana. Santiago de Chile: Comisión Económica para América Latina y el Caribe, 106 p.

[23] Kavouras. I, DuBois. I, Nikolich. G, Corral. A, Avittia, V. Etyemezian. (2015). Particulate dust emission factors from unpaved roads in the U.S.-Mexico border semi-arid region. Journal of Arid Environments, 124, pp. 189-192. doi: 10.1016/j. jaridenv.2015.07.015

[24] Claiborn.C, Mitra. A, Adams. G, Bamesberger. L, Allwine. G Kantamaneni. R, Lamb B, Westberg. H. (1995). Evaluation of PM10 emission rates from paved and unpaved roads using tracer techniques. Atmospheric Environment, 29 (10), pp. 1075-1089. doi: 10.1016/13522310(95)00046-2

[25] Venkatram. A, Fitz. D, Bumiller. K, Du. S, Boeck. M, Ganguly. C. (1999). Using a dispersion model to estimate emission rates of particulate matter from paved roads. Atmospheric Environment, 33 (7), pp. 1093-1102. doi: 10.1016/s13522310(98)00316-1

[26] Etyemezian. V, Kuhns. H, Gillies. J, Chow J, Hendrickson. K, McGown. M, Pitchford. M. (2003). Vehicle-based road dust emission measurement (III): effect of speed, traffic volume, location, and season on PM10 road dust emissions in the Treasure Valley, ID. Atmospheric Environment, 37 (32), pp. 4583-4593. doi: 10.1016/ s1352-2310(03)00530-2 